\title{
Comportamento do Algoritmo de Projeções Afins com Filtragem de Ordem Insuficiente
}

\author{
Sérgio J.M. de Almeida, Márcio H. Costa e José C. M. Bermudez
}

\begin{abstract}
Resumo - Este trabalho apresenta uma análise do algoritmo de Projeções Afins (AP) quando o tamanho do filtro adaptativo é menor que o da planta. Para tanto, assume-se a existência de um número elevado de coeficientes adaptativos (em relação à ordem do AP), sinal de entrada autoregressivo e passo de convergência unitário. Os modelos teóricos desenvolvidos permitem concluir que os coeficientes do algoritmo AP convergem, em média, para os coeficientes da planta, produzindo uma solução nãopolarizada mesmo para o caso de sinais de entrada correlacionados. Em contrapartida, o erro quadrático médio em regime permanente é adicionado de um termo proporcional à potência dos coeficientes não modelados da planta.
\end{abstract}

Palavras-Chave - Filtros adaptativos, APA, comprimento deficiente.

\begin{abstract}
This work presents a statistical analysis of the deficient length Affine Projection (AP) adaptive algorithm. It assumes a large number of adaptive coefficients (in relation to the AP order), autoregressive input signal and unity step-size. The developed theoretical models permit to conclude the AP coefficients converge, in mean, to the plant coefficients, producing an unbiased solution even for the correlated input signal case. However, the steady-state mean square error is increased by an amount proportional to the power of the nonmodeled plant coefficients.
\end{abstract}

Keywords - Adaptive filter, APA, deficient length.

\section{INTRODUÇ̃̃O}

Técnicas de filtragem adaptativa possuem amplo espectro de utilização. Dentre os diferentes algoritmos adaptativos, o algoritmo Least Mean Square (LMS) é provavelmente o mais popular, principalmente em função de seu baixo custo computacional, robustez e capacidade de rastreamento [1][2]. Em decorrência deste fato, vários autores têm estudado seu comportamento em diferentes condições de aplicação. Basicamente, para um dado valor do passo de convergência, o desempenho do LMS é determinado por dois fatores [1]-[3]: (a) o número de coeficientes adaptáveis; e (b) a estatística do sinal de entrada.

Sérgio J.M. de Almeida, Escola de Engenharia e Arquitetura, Universidade Católica de Pelotas, Pelotas-RS. Márcio H. Costa e José C. M. Bermudez, Departamento de Engenharia Elétrica, Universidade Federal de Santa Catarina, Florianópolis-SC. E-mails: smelo@eel.ufsc.br, costa@eel.ufsc.br, j.bermudez@ieee.org. Esse trabalho foi parcialmente financiado pelo $\mathrm{CNPq}$
A maioria dos estudos teóricos sobre o desempenho de filtros adaptativos considera a situação de filtragem FIR (finite impulse response) com ordem suficiente. Neste caso o número de coeficientes adaptáveis coincide com o número de parâmetros da planta. A planta é modelada por um sistema FIR e representa a parcela do sinal a ser estimado que é estatisticamente correlacionada com o sinal de referência [1][2]. Os resultados de tais estudos não se aplicam necessariamente ao caso de ordem insuficiente, bastante comum em aplicações práticas [4]. Nesses casos, o comportamento do sistema adaptável tende a se afastar do previsto pelo modelo de ordem suficiente [5]-[6].

Projetistas de sistemas adaptativos freqüentemente têm de lidar com limitações computacionais, ou com a falta de informações necessárias para determinar o comprimento do filtro com precisão. Por exemplo, a maior parte da energia da resposta ao impulso de um canal de telefonia na banda de voz está concentrada em um período de tempo relativamente curto [7]. Em aplicações de cancelamento de eco em linha telefônica, a resposta a ser identificada normalmente é esparsa, porém longa devido a um decaimento exponencial [5]. No estudo de potenciais evocados auditivos do tronco cerebral, o interesse clínico está concentrado apenas nos primeiros $10 \mathrm{~ms}$ de uma resposta ao impulso que pode durar mais de 100ms [8]-[9].

Recentemente Mayyas [10] apresentou um estudo sobre as propriedades do algoritmo LMS com filtragem de ordem insuficiente, considerando um sinal de entrada gaussiano e estacionário. Os resultados mostraram que os coeficientes adaptáveis convergem para uma solução polarizada quando o sinal de entrada é correlacionado no tempo. Essa polarização afeta não apenas o valor médio dos coeficientes, mas também o erro quadrático médio em excesso. Dessa forma, verifica-se que algoritmo LMS pode apresentar um desempenho inadequado em importantes aplicações práticas.

A análise desenvolvida por Mayyas demonstra que a polarização é conseqüência da correlação estatística que passa a existir entre o sinal de entrada e o ruído aditivo quando o sinal de entrada é correlacionado no tempo. A partir dessa constatação, é de grande interesse a busca por algoritmos adaptativos que possuam mecanismos implícitos de redução desta polarização em relação à solução ótima. Tais algoritmos são candidatos naturais para substituir o LMS em aplicações em que o uso de filtros adaptáveis de ordem insuficiente é inevitável ou simplesmente conveniente.

$\mathrm{O}$ algoritmo de Projeções Afins (AP - affine projection) [11] é conhecido por descorrelacionar o sinal de entrada durante o processo de atualização dos coeficientes. Essa 
propriedade foi demonstrada em [12] para o importante caso de passo de convergência unitário e entrada autoregressiva (AR). Assim, espera-se que o algoritmo AP possa conduzir a uma solução não-polarizada dos coeficientes adaptáveis no caso de ordem insuficiente mesmo para sinais de entrada correlacionados. Entretanto, estas características do algoritmo AP não foram demonstradas teoricamente até o momento.

O comportamento do algoritmo AP foi analisado em [13] para uma entrada AR e passo de convergência unitário, assumindo um filtro adaptável de ordem suficiente. Como resultado, é demonstrado que a ação do algoritmo AP para um sinal de entrada AR é similar à ação do algoritmo LMS normalizado com entrada branca.

Este trabalho estende a análise em [13] para o algoritmo adaptativo AP com filtragem de ordem insuficiente. Considerando uma estrutura de identificação de sistemas e um filtro adaptável com $M$ coeficientes, demonstra-se que o vetor médio dos coeficientes converge para as $M$ primeiras amostras da resposta ao impulso desconhecida. Em contrapartida, o sub-modelamento do comprimento do filtro acarreta um erro quadrático médio adicional em regime permanente que é proporcional à potência das inovações do sinal de entrada, filtrado pelos coeficientes não modelados da resposta ao impulso desconhecida.

Este artigo está organizado da seguinte maneira: a seção II introduz o modelo do sinal de entrada e a notação usada. A Seção III apresenta a equação de ajuste dos coeficientes do algoritmo AP. A Seção IV apresenta a análise estatística que resulta em modelos analíticos para o valor médio do vetor de erro dos coeficientes, o erro quadrático médio (EQM) e os momentos de segunda ordem dos coeficientes. A Seção V apresenta simulações Monte Carlo para verificar a qualidade dos modelos teóricos obtidos. Finalmente, a Seção VI apresenta as principais conclusões deste trabalho. Neste artigo, variáveis escalares são denotadas por letras maiúsculas e minúsculas, vetores são denotados por letras minúsculas em negrito e matrizes por letras maiúsculas em negrito. $\mathrm{O}$ sobrescrito $T$ denota transposição. A letra $n$ denota tempo discreto.

\section{Filtro AdAPtAtivo de COMPRIMENTo Deficiente}

Seja $u(n)$ o sinal de entrada descrito por um processo AR de ordem $P$, então

$$
u(n)=\sum_{i=1}^{P} a_{i} u(n-i)+z(n)
$$

em que $z(n)$ (a inovação) é gaussiano, branco e de média zero, com variância $\sigma_{z}^{2}$ e os $a_{i}$ são os coeficientes do processo AR. O sinal desejado $d(n)$ é relacionado com $u(n)$ através de um modelo linear na forma

$$
d(n)=\mathbf{w}_{N}^{\mathbf{o T}} \mathbf{u}_{N}(n)+r(n)
$$

em que $\mathbf{w}_{N}^{\mathbf{o}}=\left[\begin{array}{llllll}w^{o}{ }_{0} & w^{o}{ }_{1} & w^{o}{ }_{2} & \ldots & w^{o}{ }_{N-1}\end{array}\right]^{T}$ pode ser considerado como a resposta ao impulso de um sistema desconhecido com comprimento $N ; \mathbf{u}_{N}(n)=[u(n) u(n-1) u(n-2) \ldots u(n-N+1)]^{T}$ é o vetor de entrada; e $r(n)$ é um ruído aditivo, gaussiano, branco e de média zero, e independente de $u(n)$. A saída de um filtro adaptável com comprimento $M$ é dada por

$$
y(n)=\mathbf{w}^{T}(n) \mathbf{u}(n)
$$

em que $\mathbf{w}(n)=\left[w_{0}(n) w_{1}(n) w_{2}(n) \ldots w_{M-1}(n)\right]^{T} ; \mathbf{u}(n)=[u(n) u(n-$ 1) $u(n-2) \quad \ldots \quad u(n-M+1)]^{T}$ e $M<N$ (caso do comprimento deficiente). O erro instantâneo é dado por

$$
\begin{aligned}
e(n) & =d(n)-y(n) \\
& =\mathbf{w}_{N}^{\mathbf{o} T} \mathbf{u}_{N}(n)-\mathbf{w}^{T}(n) \mathbf{u}(n)+r(n) \\
& =r(n)-\mathbf{v}^{T}(n) \mathbf{u}(n)+\overline{\mathbf{w}}^{\mathbf{o} T} \overline{\mathbf{u}}(n)
\end{aligned}
$$

em que $\overline{\mathbf{w}}^{\mathbf{0}}=\left[\begin{array}{lllll}w^{o}{ }_{M} & w^{o}{ }_{M+1} & w^{o}{ }_{M+2} & \ldots & w^{o}{ }_{N-1}\end{array}\right]^{T} ; \overline{\mathbf{u}}(n)=[u(n-M) u(n-$ $M-1) u(n-M-2) \ldots u(n-N+1)]^{T}$; e $\mathbf{v}(n)=\mathbf{w}^{\mathbf{0}}{ }_{M}$-w $(n)$ é o vetor de erro dos coeficientes $\left(\mathbf{w}_{M}^{\mathbf{o}}=\left[{w^{o}}_{0} w_{1}^{o}{ }_{1} w_{2}^{o} \ldots w_{M-1}^{o}\right]^{T}\right)$. O último termo em (4) descreve a parcela do erro decorrente dos coeficientes excedentes (de número $N-M$ ) em $\mathbf{w}^{\mathbf{0}}{ }_{N}$.

\section{EQUAÇÃo DE ATUALIZAÇÃO DOS COEFICIENTES}

A equação de atualização do vetor de erro dos coeficientes do algoritmo AP, com entrada AR, pode ser escrita como [12], [13]

$$
\mathbf{v}(n+1)=\mathbf{v}(n)+\frac{\Phi(n)}{\Phi^{T}(n) \Phi(n)} e(n)
$$

em que

$$
\Phi(n)=\mathbf{u}(n)-\mathbf{U}(n) \hat{\mathbf{a}}(n)
$$

e â $(n)$ é a estimativa (obtida através do método dos mínimos quadrados) dos coeficientes do processo AR:

$$
\hat{\mathbf{a}}(n)=\left[\mathbf{U}^{T}(n) \mathbf{U}(n)\right]^{-1} \mathbf{U}^{T}(n) \mathbf{u}(n)
$$

em que $\mathbf{U}(n)=[\mathbf{u}(n-1) \mathbf{u}(n-2) \mathbf{u}(n-3) \ldots \mathbf{u}(n-P)] ; \mathbf{U}(n)^{T} \mathbf{U}(n)$ é assumida de posto $P$; e $\hat{\mathbf{a}}(n)=\left[\hat{a}_{1}(n) \hat{a}_{2}(n) \hat{a}_{3}(n) \ldots \hat{a}_{P}(n)\right]^{T}$.

Substituindo-se (4) e (6) em (5) obtém-se

$$
\begin{aligned}
& \mathbf{v}(n+1)=\mathbf{v}(n)-\frac{\Phi(n) \Phi^{T}(n)}{\Phi^{T}(n) \Phi(n)} \mathbf{v}(n) \\
& -\Phi(n)\left[\Phi^{T}(n) \Phi(n)\right]^{-1} \hat{\mathbf{a}}^{T}(n) \mathbf{U}^{T}(n) \mathbf{v}(n) \\
& +\Phi(n)\left[\Phi^{T}(n) \Phi(n)\right]^{-1}\left(r(n)+\overline{\mathbf{w}}^{\mathbf{o} T} \overline{\mathbf{u}}(n)\right)
\end{aligned}
$$

$\mathrm{Na}$ Eq. (8) pode-se verificar que o efeito de $\overline{\mathbf{w}}^{\mathbf{0}}$ é o aumento da parcela associada o ruído aditivo $r(n)$ por um termo igual a $\overline{\mathbf{w}}^{\mathbf{o} T} \overline{\mathbf{u}}(n)$. Este termo possui média zero, é correlacionado no tempo e correlacionado com o sinal $u(n)$.

\section{ANÁLISE}

Para tornar possível a obtenção dos modelos analíticos apresentados a seguir, as seguintes hipóteses estatísticas são utilizadas (para uma discussão detalhada vide [13]):

- Hipótese A1: A ordem $P$ do algoritmo AP é assumida suficiente para modelar o processo AR de entrada.

- Hipótese A2: A dependência estatística entre z $(n)$ e $\mathbf{U}(n)$ pode ser desprezada para $M>>P$.

- Hipótese A3: O vetor $\Phi(n)$ é ortogonal aos vetores coluna da matriz $\mathbf{U}(n)$.

- Hipótese A4: Os vetores $\Phi(n)$ e $\mathbf{w}(n)$ são estatisticamente independentes.

\section{A. Comportamento Médio dos Coeficientes}

Usando a Hipótese A3 $\left(\mathbf{U}^{T}(n) \Phi(n)=\mathbf{0}\right)$ pode ser mostrado 
que

$$
\left\{\begin{array}{l}
\mathbf{u}^{T}(n) \mathbf{v}(n+1)=r(n)+\overline{\mathbf{u}}^{T}(n) \overline{\mathbf{w}}^{\circ} \\
\mathbf{U}^{T}(n) \mathbf{v}(n+1)=\mathbf{U}^{T}(n) \mathbf{v}(n)
\end{array}\right.
$$

Combinando este resultado obtém-se

$$
\mathbf{U}^{T}(n) \mathbf{v}(n)=\mathbf{r}(n-1)+\overline{\mathbf{U}}^{T}(n) \overline{\mathbf{w}}^{\mathbf{0}}
$$

em que $\mathbf{r}(n-1)=[r(n-1) r(n-2) r(n-3) \ldots r(n-P)]^{T}$ e $\overline{\mathbf{U}}(n)=[\overline{\mathbf{u}}(n-$ 1) $\overline{\mathbf{u}}(n-2) \overline{\mathbf{u}}(n-3) \ldots \overline{\mathbf{u}}(n-P)]$. Substituindo (10) em (8) obtémse

$$
\begin{aligned}
& \mathbf{v}(n+1)=\mathbf{v}(n) \\
& -\frac{\Phi(n)}{\Phi^{T}(n) \Phi(n)}\left[\Phi^{T}(n) \mathbf{v}(n)-r_{a}(n)-b_{a}(n)\right]
\end{aligned}
$$

em que

$$
\left\{\begin{array}{l}
r_{a}(n)=r(n)-\hat{\mathbf{a}}^{T}(n) \mathbf{r}(n-1) \\
b_{a}(n)=\left(\overline{\mathbf{u}}^{T}(n)-\hat{\mathbf{a}}^{T}(n) \overline{\mathbf{U}}^{T}(n)\right) \overline{\mathbf{w}}^{\mathbf{0}}
\end{array}\right.
$$

Na Eq. (12), $r_{a}(n)$ corresponde ao processamento do ruído aditivo presente em $d(n)$ por um filtro de resposta finita ao impulso cujos coeficientes são idênticos aos do processo AR que gera o sinal $u(n)$. A variável $b_{a}(n)$ é determinada por um duplo processo de filtragem sobre o sinal $u(n)$. Inicialmente, pelo mesmo filtro que determina $r_{a}(n)$ e em seqüência pelos coeficientes não-modelados da planta. Isto está de acordo com os resultados obtidos em [12] e [13], os quais mostram que o algoritmo AP substitui o ruído por sua versão filtrada.

Tomando o valor esperado de (11) e usando a Hipótese A4, obtém-se

$$
\begin{gathered}
E\{\mathbf{v}(n+1)\}=\left[\mathbf{I}-E\left\{\frac{\Phi(n) \Phi^{T}(n)}{\Phi^{T}(n) \Phi(n)}\right\}\right] E\{\mathbf{v}(n)\} \\
+E\left\{\frac{\Phi(n) r_{a}(n)}{\Phi^{T}(n) \Phi(n)}\right\}+E\left\{\frac{\Phi(n) b_{a}(n)}{\Phi^{T}(n) \Phi(n)}\right\}
\end{gathered}
$$

O primeiro valor esperado em (13) já foi resolvido em [13]:

$$
E\left\{\frac{\Phi(n) \Phi^{T}(n)}{\Phi^{T}(n) \Phi(n)}\right\}=\frac{1}{G-2}
$$

em que $G=M-P$.

O segundo valor esperado em (13) é um vetor nulo, uma vez que $r(n)$ e $\Phi(n)$ são de média zero e não-correlacionados. O último valor esperado é calculado do seguinte modo

$$
\begin{aligned}
E & \left\{\Phi(n) b_{a}(n)\right\} \\
& =E\left\{\Phi(n)\left(\overline{\mathbf{u}}^{T}(n)-\hat{\mathbf{a}}^{T}(n) \overline{\mathbf{U}}^{T}(n)\right) \overline{\mathbf{w}}^{\mathbf{0}}\right\} \\
& =E\left\{\Phi(n)[\mathbf{a}-\hat{\mathbf{a}}(n)]^{T} \overline{\mathbf{U}}^{T}(n)\right\} \overline{\mathbf{w}}^{\mathbf{0}} \\
& +E\left\{\Phi(n) \overline{\mathbf{z}}^{T}(n)\right\} \overline{\mathbf{w}}^{\mathbf{0}}=\mathbf{0}
\end{aligned}
$$

Na determinação de (15) foi assumido que $\mathbf{a}(n) \cong \mathbf{a}$ (Hipótese A1) e (de (1)) $\overline{\mathbf{u}}(n)=\overline{\mathbf{U}}(n) \mathbf{a}+\overline{\mathbf{z}}(n)$. Aplicando estes resultados em (13) obtém-se uma equação determinística recursiva para o valor médio do vetor de erro dos coeficientes do algoritmo AP com comprimento deficiente

$$
E\{\mathbf{v}(n+1)\}=\frac{G-3}{G-2} E\{\mathbf{v}(n)\}
$$

Assumindo-se convergência, o valor médio do vetor de erro dos coeficientes em regime permanente pode ser obtido de (16), resultando em

$$
\lim _{n \rightarrow \infty} E\{\mathbf{v}(n)\}=\mathbf{0}
$$

A Eq. (17) demonstra que os valores médios dos coeficientes do algoritmo AP com comprimento deficiente convergem para os primeiros $M$ coeficientes da planta.

\section{B. Erro Quadrático Médio}

Substituindo-se (6) em (4) e então (10) na expressão resultante obtém-se

$$
e(n)=-\Phi^{T}(n) \mathbf{v}(n)+b_{a}(n)+r_{a}(n)
$$

Elevando-se (18) ao quadrado e avaliando-se seu valor esperado obtém-se uma expressão para o EQM

$$
\begin{aligned}
& E\left\{e^{2}(n)\right\}=\sigma_{\Phi}^{2} \operatorname{tr}\{\mathbf{K}(n)\}+E\left\{b_{a}^{2}(n)\right\}+E\left\{r_{a}^{2}(n)\right\} \\
& -2 E\left\{\Phi^{T}(n) b_{a}(n)\right\} E\{\mathbf{v}(n)\} \\
& -2 E\left\{\Phi^{T}(n) r_{a}(n)\right\} E\{\mathbf{v}(n)\}+2 E\left\{r_{a}(n) b_{a}(n)\right\}
\end{aligned}
$$

em que $E\left\{\Phi(n) \Phi^{T}(n)\right\}=\sigma_{\Phi}^{2} \mathbf{I} ; \quad \sigma_{\Phi}^{2}=\sigma_{z}^{2}(M-P) / M$ [13]; I é a matriz identidade e $\mathbf{K}(n)=E\left\{\mathbf{v}(n) \mathbf{v}^{T}(n)\right\}$.

O primeiro valor esperado em (19) pode ser avaliado usando $\overline{\mathbf{u}}(n)=\overline{\mathbf{U}}(n) \mathbf{a}+\overline{\mathbf{z}}(n)$ em (12) e assumindo $\hat{\mathbf{a}}(n) \cong \mathbf{a}$, resultando em

$$
\begin{aligned}
E\left\{b_{a}^{2}(n)\right\} & =E\left\{\left(\overline{\mathbf{u}}^{T}(n) \overline{\mathbf{w}}^{\mathbf{0}}-\hat{\mathbf{a}}^{T}(n) \overline{\mathbf{U}}^{T} \overline{\mathbf{w}}^{\mathbf{0}}\right)^{2}\right\} \\
& =\overline{\mathbf{w}}^{\mathbf{0} T} E\left\{\overline{\mathbf{z}}(n) \overline{\mathbf{z}}^{T}(n)\right\} \overline{\mathbf{w}}^{\mathbf{0}} \\
& =\sigma_{z}^{2} \overline{\mathbf{w}}^{\mathbf{0} T} \overline{\mathbf{w}}^{\mathbf{0}}
\end{aligned}
$$

A Eq. (20) demonstra que o efeito do ruído aditivo correlacionado no EQM é função da potência do sinal $z(n)$ (a inovação do sinal de entrada) filtrado pela parte não modelada da resposta ao impulso desconhecida.

O segundo valor esperado em (19) foi solucionado em [13. Eq.(32)], resultando em

$$
E\left\{r_{a}^{2}\right\}=\left(1+\mathbf{a}^{T} \mathbf{a}+\sigma_{z}^{2} \operatorname{tr}\left\{E\left\{\left[\mathbf{U}^{T}(n) \mathbf{U}(n)\right]^{-1}\right\}\right\}\right) \sigma_{r}^{2}
$$

em que $\operatorname{tr}\{\cdot\}$ é a operação traço.

O terceiro valor esperado em (19) foi obtido em (15). Os dois últimos valores esperados em (19) são nulos, uma vez que $r(n)$ é independente de $u(n)$ e $\Phi(n)$.

Usando todos os resultados obtidos em (19) obtém-se

$$
E\left\{e^{2}(n)\right\}=\left(1+\mathbf{a}^{T} \mathbf{a}\right) \sigma_{r}^{2}+\sigma_{\Phi}^{2} T_{K}(n)+\sigma_{z}^{2} \overline{\mathbf{w}}^{\circ T} \overline{\mathbf{w}}^{\mathbf{0}}
$$

em que $T_{K}(n)=\operatorname{tr}\{\mathbf{K}(n)\}$ e de [13] sabe-se que $\sigma_{z}^{2}$ $\operatorname{tr}\left\{E\left\{\left[\mathbf{U}^{T}(n) \mathbf{U}(n)\right]^{-1}\right\}\right\}<<1$ para $M>>P$. A equação recursiva para os momentos de segunda ordem $\left(T_{K}(n)\right)$ será derivada na próxima seção.

\section{Momentos de Segunda Ordem}

Os momentos de segunda ordem para o caso de comprimento deficiente podem ser obtidos da mesma forma que em [13]. Pós-multiplicando (11) por sua transposta, tomando seu valor esperado e aplicando as hipóteses estatísticas A1-A4 chega-se em 


$$
\begin{aligned}
\mathbf{K}(n+1) & =\mathbf{K}(n)-\frac{2}{(G-2)} \mathbf{K}(n) \\
& +\frac{1}{M(G+2)} \operatorname{tr}\{\mathbf{K}(n)\} \mathbf{I} \\
& +\frac{(M-G)}{G M(G+2)} E\left\{\mathbf{v}^{T}(n)\right\} E\{\mathbf{v}(n)\} \mathbf{I} \\
& +\frac{M\left(1+\mathbf{a}^{T} \mathbf{a}\right) \sigma_{r}^{2}}{G(G-2)(G-4) \sigma_{z}^{2}} \mathbf{I} \\
& +E\left\{b_{a}^{2}(n)\right\} E\left\{\frac{\Phi(n) \Phi^{T}(n)}{\left[\Phi^{T}(n) \Phi(n)\right]^{2}}\right\}
\end{aligned}
$$

em que os termos dependentes na correlação entre $b_{a}(n)$ e $\Phi(n)$ são nulos. O último valor esperado em (23) pode ser aproximado por

$$
\begin{aligned}
& E\left\{\frac{b_{a}^{2}(n) \Phi(n) \Phi^{T}(n)}{\left[\Phi^{T}(n) \Phi(n)\right]^{2}}\right\} \\
& =E\left\{b_{a}^{2}(n)\right\} E\left\{\left[\Phi^{T}(n) \Phi(n)\right]^{-2}\right\} E\left\{\Phi(n) \Phi^{T}(n)\right\} \\
& =\frac{M \overline{\mathbf{w}}^{\mathbf{o} T} \overline{\mathbf{w}}^{\mathbf{o}}}{G(G-2)(G-4)} \mathbf{I}
\end{aligned}
$$

A Eq. (25) é um modelo recursivo determinístico para o comportamento dos momentos de segunda ordem do algoritmo AP com comprimento deficiente e sinal de entrada AR. Contudo, apenas o traço de $\mathbf{K}(n)$ é necessário para determinar o EQM. Tomando o traço de (25) obtém-se

$$
\begin{aligned}
T_{K}(n+1)= & \frac{G^{2}-G-10}{(G-2)(G+2)} T_{K}(n) \\
& +\frac{(M-G)}{G(G+2)} E\left\{\mathbf{v}^{T}(n)\right\} E\{\mathbf{v}(n)\} \\
& +\frac{M^{2}\left[\left(1+\mathbf{a}^{T} \mathbf{a}\right) \sigma_{r}^{2}+\overline{\mathbf{w}}^{\mathbf{o} T} \overline{\mathbf{w}}^{\mathbf{o}} \sigma_{z}^{2}\right]}{G(G-2)(G-4) \sigma_{z}^{2}}
\end{aligned}
$$

onde $T_{K}(n)=\operatorname{tr}\{\mathbf{K}(n)\}$.

As equações (22) e (26) constituem um modelo geral para o EQM do algoritmo AP tanto para o caso do comprimento deficiente quanto para o comprimento suficiente. Os resultados apresentados em [13] podem ser considerados como um caso particular destas expressões.

\section{EQM em Regime Permanente}

Assumindo-se convergência, o EQM em regime permanente pode ser obtido de (22) como

$$
\begin{aligned}
\lim _{n \rightarrow \infty} E\left\{e^{2}(n)\right\} & =\left(1+\mathbf{a}^{T} \mathbf{a}\right) \sigma_{r}^{2}+\sigma_{z}^{2} \overline{\mathbf{w}}^{\mathbf{o T}} \overline{\mathbf{w}}^{\mathbf{o}} \\
& +\sigma_{\Phi}^{2} \lim _{n \rightarrow \infty} T_{K}(n)
\end{aligned}
$$

em que, de (26)

$$
\lim _{n \rightarrow \infty} T_{K}(n)=\frac{M^{2}(G+2)\left[\left(1+\mathbf{a}^{T} \mathbf{a}\right) \sigma_{r}^{2}+\overline{\mathbf{w}}^{\mathbf{o} T} \overline{\mathbf{w}}^{\mathbf{0}} \sigma_{z}^{2}\right]}{G(G+6)(G-4) \sigma_{z}^{2}}
$$

Substituindo-se (28) em (27) obtém-se

$$
\lim _{n \rightarrow \infty} E\left\{e^{2}(n)\right\}=k\left(1+\mathbf{a}^{T} \mathbf{a}\right) \sigma_{r}^{2}+k \overline{\mathbf{w}}^{\mathbf{o} T} \overline{\mathbf{w}}^{\mathbf{0}} \sigma_{z}^{2}
$$

em que

$$
k=\left[1+\frac{M(G+2)}{(G+6)(G-4)}\right]
$$

\section{SIMULAÇ̃̃ES}

Esta seção apresenta resultados de simulações com a finalidade de verificar a qualidade dos modelos analíticos definidos em (22), (26) e (29). Simulações Monte Carlo da Eq. (5) permitem verificar uma excelente acurácia com a expressão teórica dada pela Eq. (16) (Figura 2). Os exemplos apresentados assumem uma planta que emula um caminho de eco com uma constante de decaimento [14] com 256 coeficientes (Figura 1) e uma relação sinal-ruído (SNR signal to noise ratio) de $60 \mathrm{~dB}$. O sinal $z(n)$ tem potência $\sigma_{z}^{2}=0.19$ para o modelo AR de primeira ordem (Figura 3) e $\sigma_{z}^{2}=0.6$ para os casos dos modelos AR de segunda e terceira ordem (Figuras 4 e 5). O ruído aditivo $(r(n))$ tem potência $\sigma_{r}^{2}=10^{-6}$ para todos os exemplos. A inicialização dos coeficientes adaptativos foi realizada na origem $\mathbf{w}(0)=\mathbf{0}$ e um fator de regularização de $10^{-4}$ foi adicionado aos elementos da diagonal de $\mathbf{U}^{T}(n) \mathbf{U}(n)$ antes da obtenção de sua inversa. As simulações Monte Carlo correspondem à média de 400 realizações.

Nas Figuras 3 até 5 são apresentadas curvas do EQM obtidas para diferentes conjuntos de parâmetros que são informados no canto superior direito de cada figura. Nessas figuras, $N$ é o número de coeficientes na planta, $M$ é o número de coeficientes do filtro adaptável, $P$ é a ordem do algoritmo AP e a é o vetor com os coeficientes do processo AR. Cada figura apresenta uma comparação entre simulações (curvas hachuradas em amarelo), modelo teórico (linha preta - Eq. (22)) e o modelo teórico para o EQM em regime permanente (linha horizontal - Eq. (29)). Os valores de $M$ iguais a 30, 40, 50, 60 e 70 em cada figura correspondem, respectivamente, às concentrações de $61,68,84,87$ e $88 \%$ do total da potência da resposta ao impulso nas primeiras $M$ amostras $\left(\mathbf{w}^{\mathbf{0} T} \mathbf{w}^{\mathbf{0}}=1\right.$ em todos os casos $)$. Todos os exemplos apresentam um excelente casamento entre os resultados teóricos e simulações Monte Carlo. 


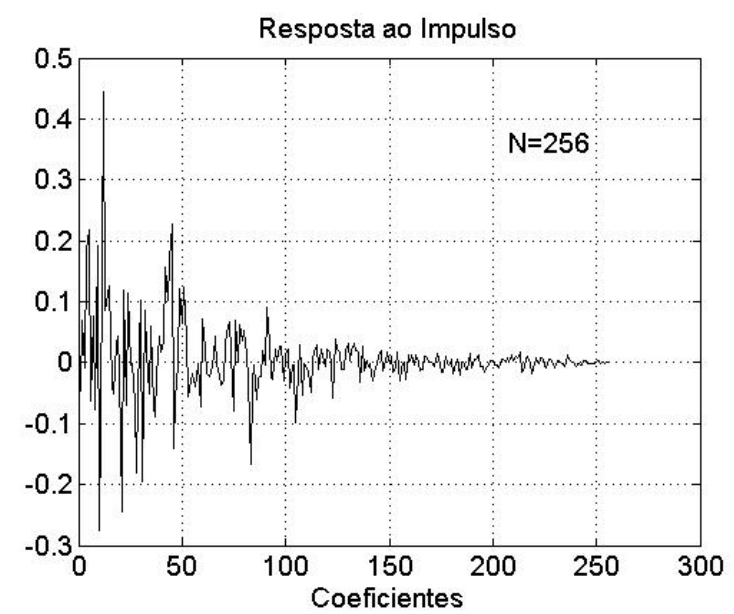

Fig. 1. Resposta ao impulso da planta

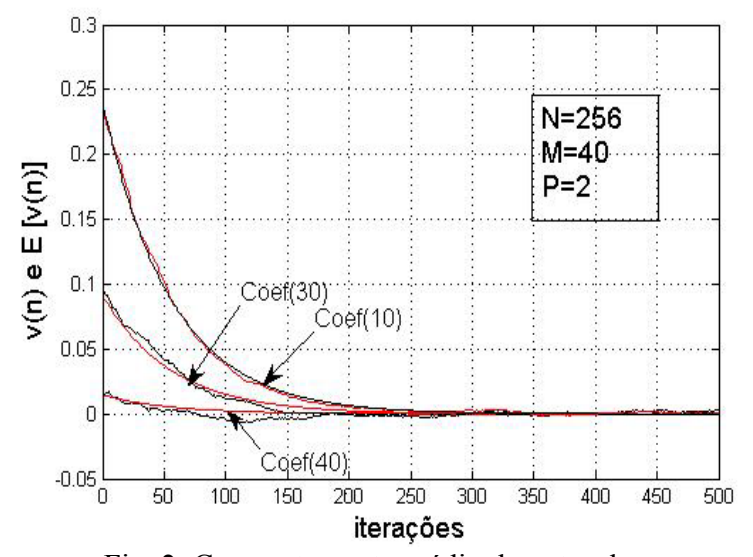

Fig. 2. Comportamento médio do vetor de erro de coeficientes

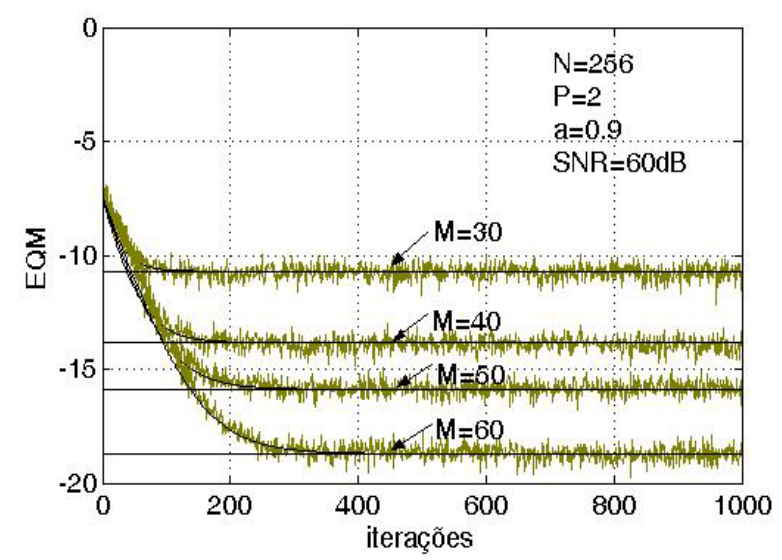

Fig. 3. Evolução do EQM para o algoritmo AP deficiente

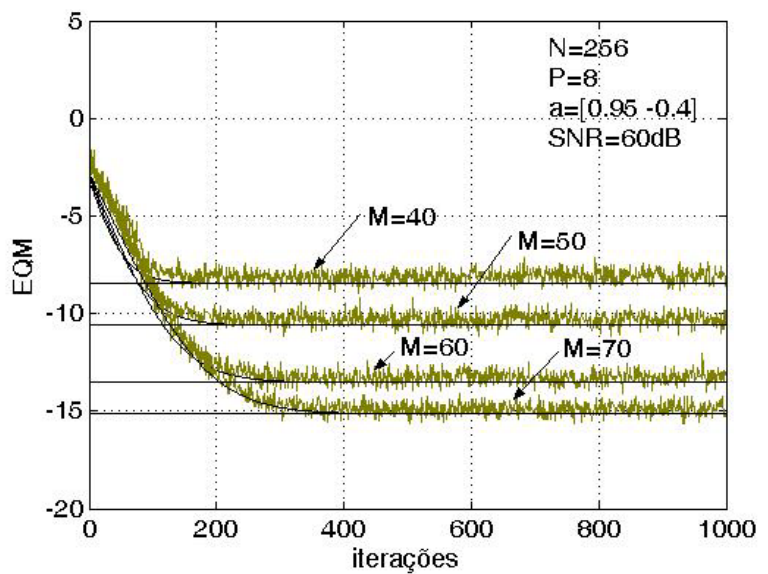

Fig. 4. Evolução do EQM para o algoritmo AP deficiente

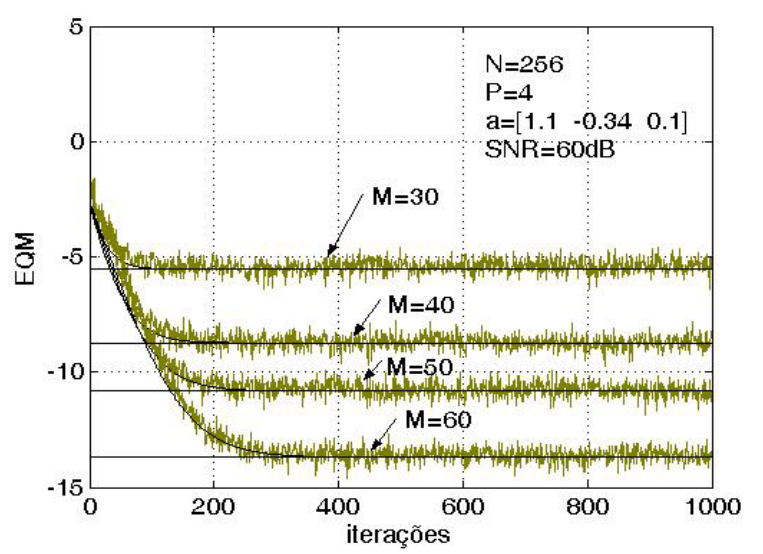

Fig. 5. Evolução do EQM para o algoritmo AP deficiente

\section{CONCLUSÕES}

Este trabalho apresenta uma análise teórica do algoritmo adaptativo de Projeções Afins que inclui a possibilidade de uma filtragem adaptável de ordem insuficiente. Equações recursivas determinísticas foram obtidas para o valor médio dos coeficientes e para o erro quadrático médio, assumindo um elevado número de coeficientes adaptáveis (comparado com a ordem do algoritmo), sinal de entrada autoregressivo, e passo de adaptação unitário. Dois resultados principais foram obtidos. Em primeiro lugar foi demonstrado que os coeficientes do algoritmo AP convergem na média para os coeficientes iniciais da planta, produzindo uma solução nãopolarizada mesmo para o caso do sinal de entrada correlacionado. Em segundo lugar foi demonstrado que o erro quadrático médio em regime permanente é adicionado de um termo proporcional à potência do sinal de inovação do sistema $\mathrm{AR}$ ( $\operatorname{sinal} z(n))$ filtrado pela parcela não modelada da resposta ao impulso desconhecida. Simulações Monte Carlo mostraram um excelente casamento com os dados obtidos a partir da predição teórica. Estes resultados reforçam a idéia conceitual de que o algoritmo AP pode ser uma boa alternativa comparado ao LMS em problemas de identificação em que o sub-modelamento da planta é esperado ou desejado. 


\section{REFERÊNCIAS}

[1] S. Haykin, Adaptive Filter Theory. Prentice-Hall, 1991.

[2] D. G. Manolakis, V. K. Ingle, and S. M. Kogon, Statistical and Adaptive Signal Processing: Spectral Estimation, Signal Modeling, Adaptive Filtering and Array Processing. Artech House Publishers, 2000.

[3] J. Homer, R. R. Bitmead, and I. Mareels, "Quantifying the effects of dimension on the convergence rate of the LMS adaptive FIR estimator," IEEE Transactions on Signal Processing, vol. 46, no. 10, pp. 2611-2615, Oct. 1998.

[4] R. C. Bilcu, P. Kuosmanen, and K. Egiazarian, "A new variable length LMS algorithm: theoretical analysis and implementations," in IEEE Int. Conference on Electronics, Circuits and Systems, Dubrovnik, Croatia, September 15-18. 2002, pp. 10311034.

[5] Y. Gu, K. Tang, and H. Cui., "LMS algorithm with gradient descent filter length," IEEE Signal Processing Letters, vol. 11, no. 3, pp. 305-307, Mar. 2004.

[6] Y. Gong, and C. F. N. Cowan, "An LMS style variable taplength algorithm for structure adaptation," IEEE Transactions on Signal Processing, vol. 53, no. 7, pp. 2400-2407, Jul. 2005.

[7] K. Wesolowski, C. M. Zhao, and W. Rupprecht, "Adaptive LMS transversal filters with controlled length," IEE Proceedings- $F$, vol. 139, no. 3, pp. 233-238, Jun. 1992.

[8] K. H. Chiappa, Evoked Potentials in Clinical Medicine, Lippincot-Raven Publishers, 1997.

[9] P. Laguna, et al., "Adaptive filter for event-related bioelectric signals using an impulse correlated reference input: comparison with signal averaging techniques," IEEE Transactions on Biomedical Engineering, vol. 39, no. 10, pp. 1032-1044, Oct. 1992.

[10] K. Mayyas, "Performance analysis of the deficient length LMS adaptive filter," IEEE Transactions on Signal Processing, vol. 53, no. 8, pp. 2727-2734, Aug. 2005.

[11] K. Ozeki and T. Umeda, "An adaptive filtering algorithm using orthogonal projection to an affine subspace and its properties," Electron. Commun. Jpn., vol 67-A, no.5, pp. 19-27, Feb. 1984.

[12] M. Rupp, "A family of filter algorithms with decorrelating properties," IEEE Transactions on Signal Processing, vol. 46, no. 3, pp. 771-775, Mar. 1998 .

[13] S. J. M. Almeida, J. C. M. Bermudez, N. J. Bershad, and M. H. Costa, "A statistical analysis of the Affine Projection algorithm for unity step size and autoregressive inputs," IEEE Transactions on Circuits and Systems - I, vol. 52, no. 7, pp. 1394-1405, Jul. 2005.

[14] Y. Gu, K. Tang, H. Cui, and W. Du, "Convergence analysis of a deficient-length LMS filter and optimal-length sequence to model exponential decay impulse response," IEEE Signal Processing Letters, vol. 10, no. 1, pp. 4-7, Jan. 2003. 